\title{
Editorial
}

\section{Leadership role of pediatric surgeon during COVID-19 Pandemic}

\author{
Sameh Shehata \\ Emeritus Professor of Pediatric Surgery, University of Alexandria Children's Hospital \\ President World Federation of Associations of Pediatric Surgeons (WOFAPS)
}

\begin{abstract}
Cite as: Shehata S. Leadership role of pediatric surgeon during COVID-19 Pandemic. J Pediatr Adolesc Surg. 2020 ; 1:1.
This is an open-access article distributed under the terms of the Creative Commons Attribution License, which permits unrestricted use, distribution, and reproduction in any medium, provided the original work is properly cited (https://creativecommons.org/ licenses/by/4.0/).
\end{abstract}

The outbreak of COVID-19 has led to enormous challenges in the medical field in all specialties. It required prompt and effective measures to confront the global pandemic. In pediatric surgery like other specialties, it has implications and responsibilities on pediatric surgeons towards their patients, team members, and community at large. The COVID-19 outbreak led to almost complete postponement of elective operations as well as minimally invasive and endoscopic surgeries. This results a huge back log of cases waiting for their surgery. This calls for a leadership role of pediatric surgeons to organize the waiting lists by prioritizing the cases based on need or urgency and also ensuring safety of healthcare workforce and patients. Maximizing efforts, necessary changes in OR and staffing are necessary to finish this backlog in a reasonable time, as a setup is getting ready to resume elective surgery.

While elective cases have been postponed, the pediatric surgeons still have the responsibility to operate on emergency cases and tumors. It is huge responsibility of the pediatric surgeon to ensure his own safety as well as the safety of the team members including, anesthetists, nurses and assistants.
There are many published guidelines on the safe procedures in the OR and outpatient clinics with the use of PPE and safe procedures. The pediatric surgeon is responsible to convey updated information about safety and protection to his patients and their parents including postponing elective surgery while taking the necessary precaution during outpatient clinics including minimum accompanying persons, the proper use of masks and social distancing. Online consultation is a great way of reducing physical contact while maintaining good communication between the patients and their treating doctors for follow-up on operated cases or for taking advice or appointments for preoperative cases. Online webinars are a great tool for spreading updated information by experts in pediatric surgery to young doctors in remote areas.

There are many lessons and experiences we have learned from this hardship, I am sure that we are going to overcome this difficult time soon with the excellent and heroic acts of pediatric surgeons around the globe, who have done great efforts and risk their lives to maintain their role in treating and protecting their patients. 